\title{
SMALLEST CUBIC AND QUARTIC GRAPHS WITH A GIVEN NUMBER OF CUTPOINTS AND BRIDGES
}

\author{
GARY CHARTRAND and FARROKH SABA \\ Department of Mathematics, Western Michigan University \\ Kalamazoo, Michigan 49008 U.S.A. \\ JOHN K. COOPER, JR. \\ Department of Mathematics, Eastern Michigan University \\ Ypsilanti, Michigan 48197 U.S.A. \\ FRANK HARARY \\ Department of Mathematics, University of Michigan \\ Ann Arbor, Michigan 48104 U.S.A.
}

CURTISS E. WALL

Department of Mathematics, Old Dominion University Norfolk, Virginia 23508 U.S.A.

(Received April 6, 1981)

ABSTRACT. For positive integers $b$ and $c$, with $c$ even, satisfying the inequalities $b+1 \leq c \leq 2 b$, the minimum order of a connected cubic graph with $b$ bridges and c cutpoints is computed. Furthermore, the structure of all such smallest cubic graphs is determined. For each positive integer $c$, the minimum order of a quartic graph with c cutpoints is calculated. Moreover, the structure and number of all such smallest quartic graphs are determined.

KEY WOKDS AND PHRASES. Cutpoints, Bridges, Cubic graphs, Quartic graphs. 1980 MATHEMATICS SUBJECT CLASSIFICATION CODES. O5C99

1. INTRODUCTION.

Eitner and Harary [1] showed that if $G$ is a connected cubic graph with $b(\geq 1)$ bridges and $c(\geq 1)$ cutpoints, then $c$ is even and $b+1 \leq c \leq 2 b$. They further showed that for positive integers $b$ and $c$, with $c$ even, satisfying $b+1 \leq c \leq 2 b$, 
there exists a connected cubic graph with b bridges and c cutpoints. We determine the smallest order of such cubic graphs as well as their structure.

Graphs having only vertices of even degree do not contain bridges (their components are eulerian). Thus every quartic (4-regular) graph is bridgeless. For every positive integer $c$, the minimum order of a quartic graph with c cutpoints is computed. The structure and number of all such smallest quartic graphs are determined.

Throughout the paper we follow [2] and [3] for basic terminology in graph theory.

2. SMALLEST CUBIC GRAPHS WITH A GIVEN NUMBER OF CUTPOINTS AND BRIDGES.

THEOREM 2.1. Let $b$ and $c$ be positive integers, with $c$ even, such that $b+1 \leq c \leq 2 b$. Then the minimum order of a connected cubic graph with $b$ bridges and c cutpoints is $2 b+c+6$.

PROOF. Among the connected cubic graphs having $b$ bridges and $c$ cutpoints, let $\mathrm{G}$ be one of minimum order p. Let $\mathrm{k}(0 \leq \mathrm{k} \leq \mathrm{c})$ be the number of cutpoints incident with exactly one bridge; then, of course, $G$ has $c-k$ cutpoints incident with three bridges.

Let $\mathrm{n}$ be the number of blocks in $G$. Also, for $v \in V(G)$, denote the number of blocks of $\mathrm{G}$ containing $\mathrm{v}$ by $\mathrm{n}(\mathrm{v})$ (the number $\mathrm{n}(\mathrm{v})$ is sometimes called the block index of $v)$. Thus, $n(v)=1$ if $v$ is not a cutpoint, $n(v) \equiv 2$ if $v$ is a cutpoint incident with exactly one bridge and $n(v)=3$ if $v$ is a cutpoint incident with three bridges.

Using the elementary formula of [4],

$$
\mathrm{n}=1+\sum_{\mathrm{v} \in \mathrm{V}(\mathrm{G})}[\mathrm{n}(\mathrm{v})-1],
$$

we see that $n=1+2 k+3(c-k)-c$ or

$$
\mathrm{n}=2 \mathrm{c}-\mathrm{k}+1 \text {. }
$$

If we sum the number of bridges incident with a cutpoint over all cutpoints, we obtain $2 b=3(c-k)+k$ so that

$$
k=(3 c-2 b) / 2 .
$$


Substituting the expression for $\mathrm{k}$ in (2.2) into (2.1), we obtain

$$
\mathrm{n}=1+\mathrm{b}+\mathrm{c} / 2 \text {. }
$$

Thus, among the $1+b+c / 2$ blocks of $G$, the $b$ bridges are acyclic and $1+c / 2$ are cyclic.

An end-block of $G$ necessarily has one vertex of degree 2 and all others of degree 3. The smallest block with this property has order 5 and is the unique block $B_{1}$ of Figure 1. Necessarily, then, every end-block of $G$ is isomorphic to $\mathrm{B}_{1}$

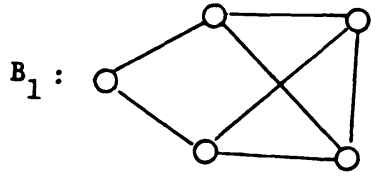

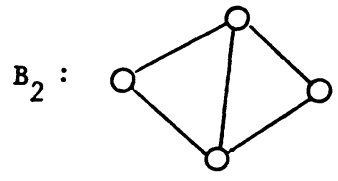
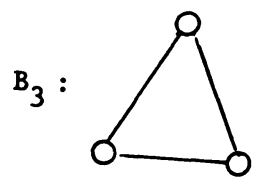

Figure 1

In a cubic graph the smallest cyclic block with exactly two cutpoints is isomorphic to the block $B_{2}$ of Figure 1 while the smallest block with exactly three cutpoints is isomorphic to the block $B_{3}$ of Figure 1 . Indeed, any block of $G$ having exactly $i$ cutpoints $(i \geq 3)$ must be isomorphic to the cycle $C_{i}$ Thus, if a cyclic block of $G$ contains exactly $i$ cutpoints, then the block is isomorphic to $B_{i}$ if $i=1,2$ and is isomorphic to $c_{i}$ if $i \geq 3$. For $i \geq 1$, let $n_{i}$ denote the number of cyclic blocks containing exactly $i$ cutpoints of $G$. Thus,

$$
\mathrm{n}=\mathrm{b}+\sum_{i=1} \mathrm{n}_{i}
$$

We may assume that $\mathrm{n}_{3}=0$; for if $\mathrm{n}_{3}>0$, then $G$ can be transformed into a cubic graph $\mathrm{G} *$ of order $\mathrm{p}$ having b bridges, $\mathrm{c}$ cutpoints and $\mathrm{n}_{3}=0$. In order to see this, let B be a block of $G$ having order 3 and let A be an end-block of $G$ (see Figure 2). Let $u$ be the cutpoint of $G$ in $A$, and let $G^{\prime}$ be the graph obtained from $G$ by deleting the edges of $B$ and the non-cutpoints of $A$. Let $H$ be the component of $G^{\prime}$ containing $u$, and let $v$ be the vertex of $H$ that belongs to $B$. Let $w_{1}$ and $w_{2}$ be the other two vertices of $B$, where $H_{i}(i=1,2)$ is the component of $\mathrm{G}^{\prime}$ containing $\mathrm{w}_{i}{ }^{\bullet}$ 


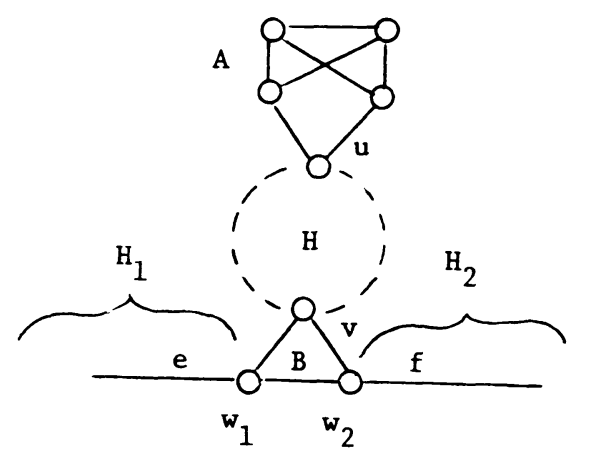

G
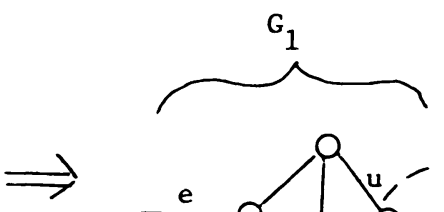

Figure 2

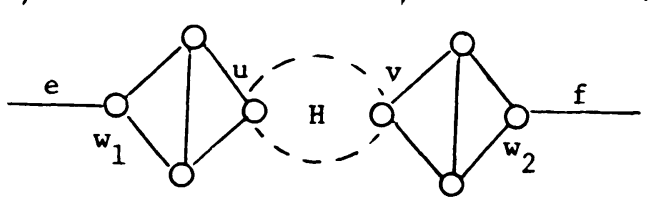

$G_{1}^{*}$

For $i=1,2$, the graph $G_{i}$ is defined by identifying a vertex of degree 2 of the block $B_{2}$ (of Figure 1) with the vertex $w_{i}$ of $H_{i}$. The graph $G_{1}{ }^{*}$ is now obtained by identifying the vertex $\mathrm{u}$ of $\mathrm{H}$ with the vertex of degree 2 in $\mathrm{G}$, and identifying $\mathrm{v}$ with the vertex of degree 2 in $G_{2}$ (see Figure 2). The cubic graph $G_{1}{ }^{*}$ has $n_{3}-1$ blocks isomorphic to $\mathrm{C}_{3}$ and has order $\mathrm{p}, \mathrm{b}$ bridges and c cutpoints. Continuing this procedure, if necessary, we arrive at the desired cubic graph $G^{*}$. Thus we can assume that $\mathrm{n}_{3}=0$ for the graph $G$.

Next we show that $n_{i}=0$ for all $i \geq 4$. Suppose that $n_{j}>0$ for some $j \geq 4$. Then $G$ contains a cycle $c_{j}: w_{1}, w_{2}, \ldots w_{j}$, $w_{1}$, where then each $w_{i}(1 \leq i \leq j)$ is a cutpoint of $G$. Let $F$ be the graph obtained by deleting the edges of $C_{j}$ from $G$, and 1 et $F_{i}(1 \leq i \leq j)$ be the component of $F$ containing $w_{i}$.

Let $\hat{B}$ be an end-block of $G$ that is also a block of $F_{1}$, and let $w_{0}$ be the cutpoint of $G$ belonging to $\hat{B}$. Note that $\hat{B}$ is isomorphic to the block $B_{1}$ of Figure 1 . We construct the graph $\hat{G}$ by (1) deleting the edges $w_{j-1} w_{j}$ and $w_{j} w_{1}$ from $G$, (2) adding the edge $w_{1} w_{j-1}$, (3) deleting all vertices of $\hat{B}$ from $G$ except $w_{0}$, (4) identifying one vertex of degree 2 in the block $B_{2}$ of Figure 1 with $w_{0}$, and (5) identifying the other vertex of degree 2 in $B_{2}$ with the vertex $w_{j}$ of $F_{j}$. The graph $\hat{G}$ is cubic and has $\mathrm{b}$ bridges, $\mathrm{c}$ cutpoints and order $\mathrm{p}-2$; however, this is impossible due to the manner in which $G$ was chosen. Hence we may assume that $n_{i}=0$ for $i \geq 3$.

The expression (2.4) for the number $n$ of blocks of $G$ can now be written as

$$
\mathrm{n}=\mathrm{b}+\mathrm{n}_{1}+\mathrm{n}_{2}
$$


It follows (by considering the block-cutpoint tree (see [5]) of G for example) that the number $n_{1}$ of end-blocks of $G$ is given by

$$
\mathrm{n}_{1}=2+(\mathrm{c}-\mathrm{k})=2+\mathrm{b}-\mathrm{c} / 2 \text {, }
$$

where the latter expression for $\mathrm{n}_{1}$ is a consequence of (2.2). By combining $(2.3),(2.5)$ and $(2.6)$, we have

$$
\mathrm{n}_{2}=\mathrm{n}-\mathrm{n}_{1}-\mathrm{b}=(1+\mathrm{b}+\mathrm{c} / 2)-(2+\mathrm{b}-\mathrm{c} / 2)-\mathrm{b}=\mathrm{c}-1-\mathrm{b} \text {. }
$$

Thus, (2.6) and (2.7) imply that the order $p$ of $G$ is

$p=(c-k)+5 n_{1}+4 n_{2}=(b-c / 2)+5(2+b-c / 2)+4(c-1-b)=2 b+c+6$.

By the preceding proof, it follows that each cyclic block of a connected cubic graph having b bridges, $c$ cutpoints and order $2 b+c+6$ is isomorphic to one of $B_{1}, B_{2}$ (of Figure 1 ) and $C_{3}$ and, conversely, if $G$ is a connected cubic graph with $b$ bridges and c cutpoints, every cyclic block of which is isomorphic to $B_{1}$, $B_{2}$ or $C_{3}$, then $G$ has order $2 b+c+6$. It therefore follows that the construction of Eitner and Harary [1] of a connected cubic graph with b bridges and $c$ cutpoints for $\mathrm{all} b$ and $c$, with $c$ even, and $b+1 \leq c \leq 2 b$ has, in fact, the minimum possible order, namely $2 b+c+6$.

\section{SMALLEST QUARTIC GRAPHS WITH A GIVEN NUMBER OF CUTPOINTS}

THEOREM 3.1. For a positive integer $c$, the minimum order of a quartic graph with c cutpoints is $(7 c+15) / 2$ if $c$ is odd and $(7 c+18) / 2$ if $c$ is even.

PROOF. Among the quartic graphs with $c$ cutpoints, let $G$ be one of minimum order $\mathrm{p}$. Suppose that $\mathrm{G}$ has $\mathrm{n}$ blocks, and let $\mathrm{n}(\mathrm{v})$ denote the number of blocks containing a vertex $\mathrm{v}$ of $\mathrm{G}$. If $\mathrm{v}$ is not a cutpoint, then $\mathrm{n}(\mathrm{v})=1$; while if $\mathrm{v}$ is a cutpoint, then $n(v)=2$. As mentioned in the proof of Theorem 2.1,

$$
\mathrm{n}=1+\sum_{\mathrm{v} \in \mathrm{V}(\mathrm{G})}[\mathrm{n}(\mathrm{v})-1] \text {. }
$$

Hence it follows that

$$
\mathrm{n}=1+\mathrm{c} \text {. }
$$

Let $n_{i}(1 \leq i \leq c)$ be the number of blocks of $G$ containing exactly $i$ cutpoints. Thus

$$
\sum_{i=1}^{\infty} \text { in }_{i}=2 \mathrm{c} .
$$


Every end-block of $G$ is necessarily isomorphic to the block $E_{1}$ of Figure 3 and every block of $G$ containing exactly two cutpoints is isomorphic to the block $E_{2}$ of Figure 3. Note that each of $E_{1}$ and $E_{2}$ has order 6 . Moreover, each block of $G$ containing exactly $i$ cutpoints $(i \geq 3)$ is isomorphic to the cycle $c_{i}$.
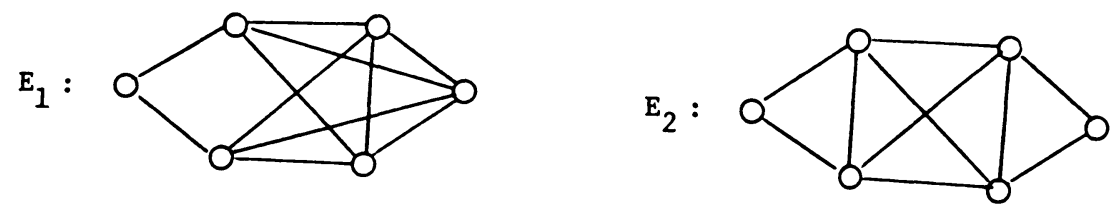

Figure 3

By an argument completely analogous to the proof of Theorem 2.1 , it can be shown that $n_{i}=0$ for all $i \geq 4$. Hence expressions (3.1) and (3.2) become

$$
\mathrm{n}_{1}+\mathrm{n}_{2}+\mathrm{n}_{3}=1+\mathrm{c}
$$

and

$$
n_{1}+2 n_{2}+3 n_{3}=2 c
$$

respectively. Moreover, the order of $G$ is

$$
p=6 n_{1}+6 n_{2}+3 n_{3}-c .
$$

Eliminating $\mathrm{n}_{1}$ in (3.3) and (3.4), we obtain

$$
\mathrm{n}_{3}=\left(\mathrm{c}-1-\mathrm{n}_{2}\right) / 2 \text {. }
$$

With the aid of (3.3) and (3.6), the expression for $p$ in (3.5) becomes

$$
\mathrm{p}=\left(7 \mathrm{c}+15+3 \mathrm{n}_{2}\right) / 2 \text {. }
$$

Expression (3.7) implies that $p \geq(7 c+15) / 2$. For $c$ odd we construct a quartic graph $G$, with $c$ cutpoints and order $(7 c+15) / 2$, implying that $(7 c+15) / 2$ is the minimum order of such a graph and that $\mathrm{n}_{2}=0$ in such graphs. If $c=1$, then $G_{1}$ is the graph of order 11 shown in Figure 4 . Suppose that $c=2 k+1 \geq 3$. Let $B_{1}, B_{2}, \ldots, B_{k}$ be $k$ blocks isomorphic to $C_{3}$, where $B_{1}$ and $B_{1}+1(1 \leq 1 \leq k-1)$ have a cutpoint in common. Also, every vertex belonging to exactly one $B_{j}(1 \leq j \leq k)$ 
also belongs to a block isomorphic to $E_{1}$. The graph so constructed is $G_{1}$. Thus $G_{1}$ has $k$ blocks isomorphic to $\mathrm{C}_{3}, \mathrm{k}+2$ blocks isomorphic to $\mathrm{E}_{1}, 2 \mathrm{k}+1$ (=c) cutpoints and order $(7 c+15) / 2$.

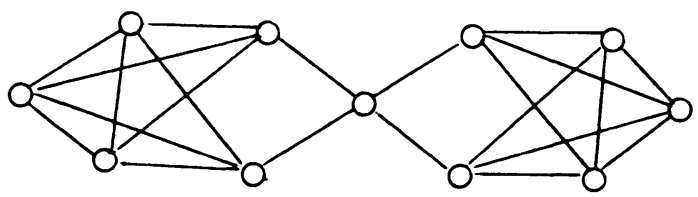

Figure 4

If $c$ is even, then (3.7) implies that $n_{2} \geq 1$ and $p \geq(7 c+18) / 2$. We construct a quartic graph $G_{2}$ with $c$ cutpoints and order $(7 c+18) / 2$, thereby verifying that this is the minimum order and, further, that $\mathrm{n}_{2}=1$. Let $c=2 \mathrm{~m} \geq 2$. If $\mathrm{c}=2$, take $G_{2}$ to be the graph of Figure 5. Otherwise, let $G_{2}^{\prime}$ be the graph having $m-1$ blocks $B_{1}, B_{2}$, . . , $B_{m-1}$, each isomorphic to $C_{3}$, where $B_{i}$ and $B_{i+1}(1 \leq i \leq m-2)$ have a cutpoint in common. Let $w$ be a vertex of degree 2 in $G_{2}^{\prime}$. Identify with $w$ a vertex of degree 2 in a block isomorphic to $\mathrm{E}_{2}$. The remaining vertex of degree 2 in $E_{2}$ is identified with the vertex of degree 2 in a block isomorphic to $E_{1}$. Every other vertex of degree 2 in $G_{2}^{\prime}$ is identified with the vertex of degree 2 in a block isomorphic to $E_{1}$. The resulting graph is $G_{2}$, which then has $\mathrm{m}-1$ blocks isomorphic to $\mathrm{C}_{3}, \mathrm{~m}+1$ blocks isomorphic to $\mathrm{E}_{1}$, one block isomorphic to $\mathrm{E}_{2}$, $2 \mathrm{~m}$ (=c) cutpoints and order $(7 c+18) / 2$.

The preceding proof also has the following corollary.

COROLLARY. For each odd positive integer $c$, there is exactly one quartic graph with c cutpoints and having order $(7 c+15) / 2$. For each even positive integer $c$, there are exactly [c/4] quartic graphs with c cutpoints and order $(7 c+18) / 2$ if $c \geq 4$ and exactly one such quartic graph if $c=2$.

The problem of determining the number of (non-isomorphic) connected cubic graphs with $\mathrm{b}$ bridges, $\mathrm{c}$ cutpoints $(\mathrm{b}+1 \leq \mathrm{c} \leq 2 \mathrm{~b})$ and order $2 \mathrm{~b}+\mathrm{c}+6$ remains unsettled. 


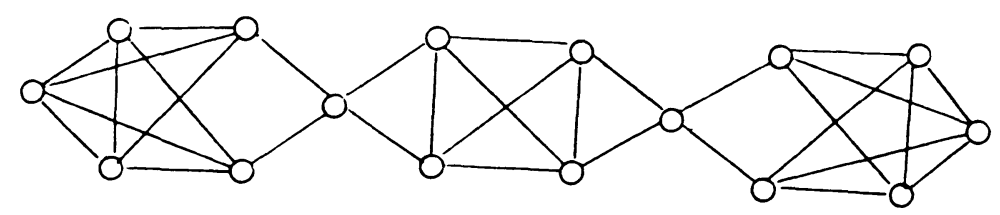

Figure 5

\section{REFERENCES}

1. EITNER, P.G. and HARARY, F. The Number of Bridges and Cutpoints in a Cubic Graph, Pi Mu Epsilon J. I(1981), 242-247.

2. BEHZAD, M., ChARTRAND, G. and LESNIAK-FOSTER, L. Graphs \& Digraphs. Prindle Weber \& Schmidt, Boston (1979).

3. HARARY, F. Graph Theory. Addison-Wesley, Reading (1969).

4. HARARY, F. An Elementary Theorem on Graphs, Amer. Math. Month1y, 66 (1959), 405-407.

5. HARARY, F. and PRINS, G. The Block-Cutpoint Tree of a Graph, Publ. Math Debrecen, 13 (1966), 103-107. 


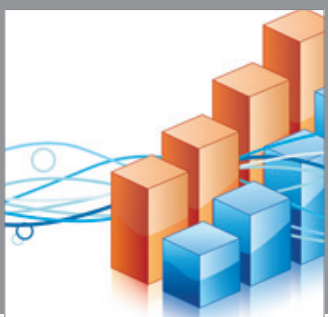

Advances in

Operations Research

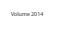

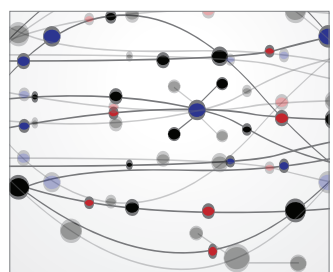

\section{The Scientific} World Journal
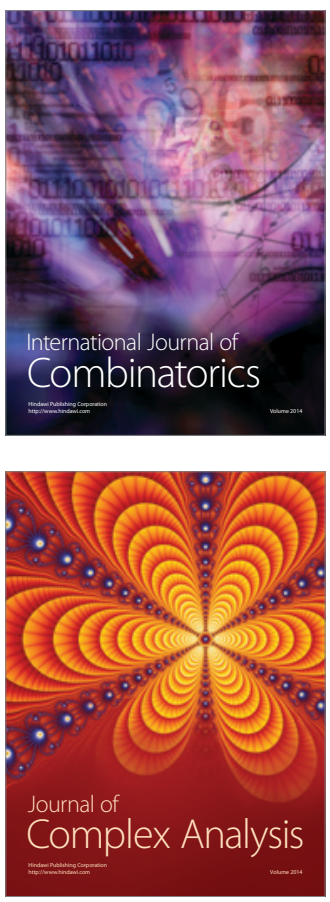

International Journal of

Mathematics and

Mathematical

Sciences
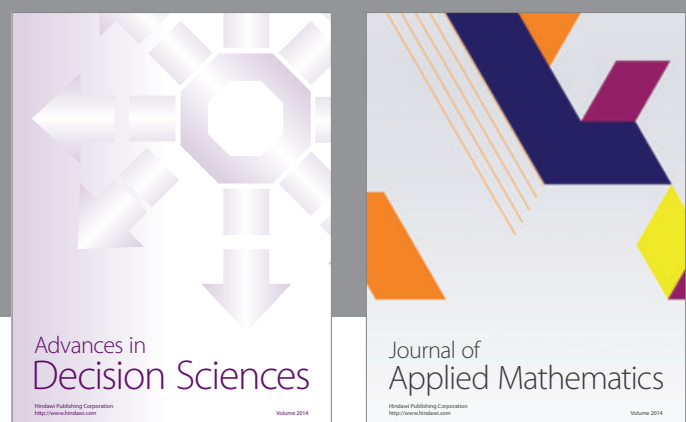

Journal of

Applied Mathematics
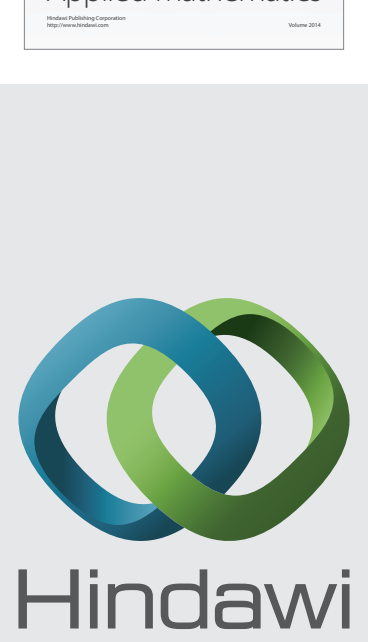

Submit your manuscripts at http://www.hindawi.com
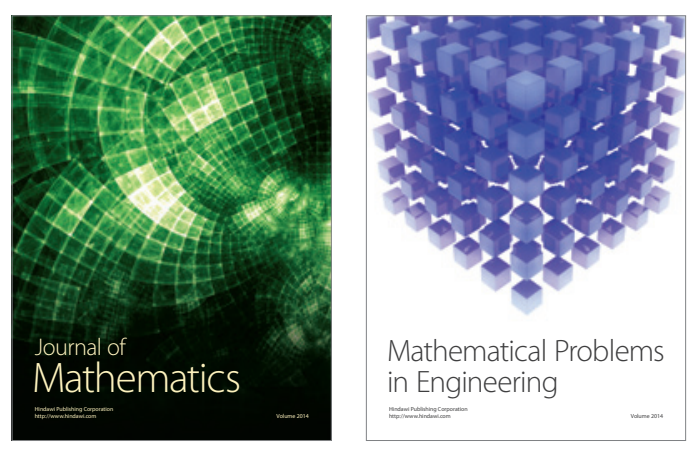

Mathematical Problems in Engineering
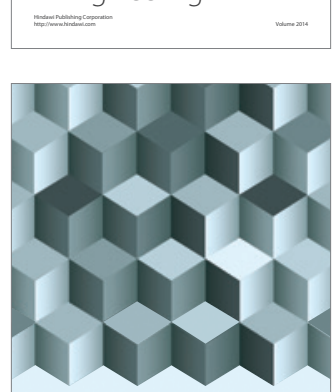

Journal of

Function Spaces
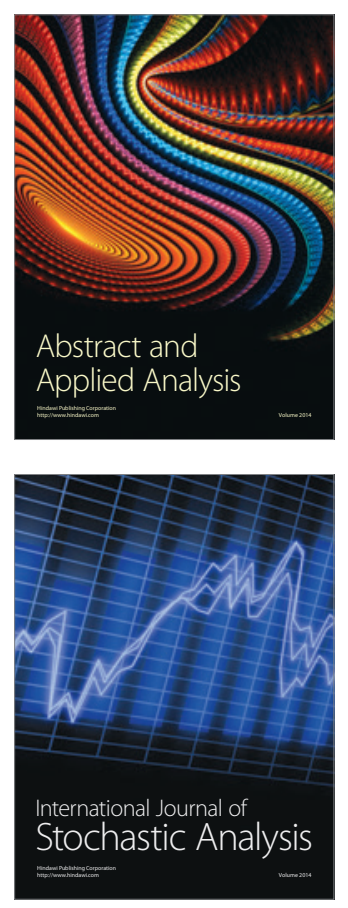

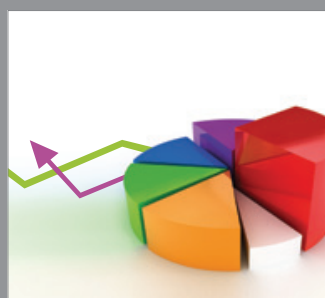

ournal of

Probability and Statistics

Promensencen
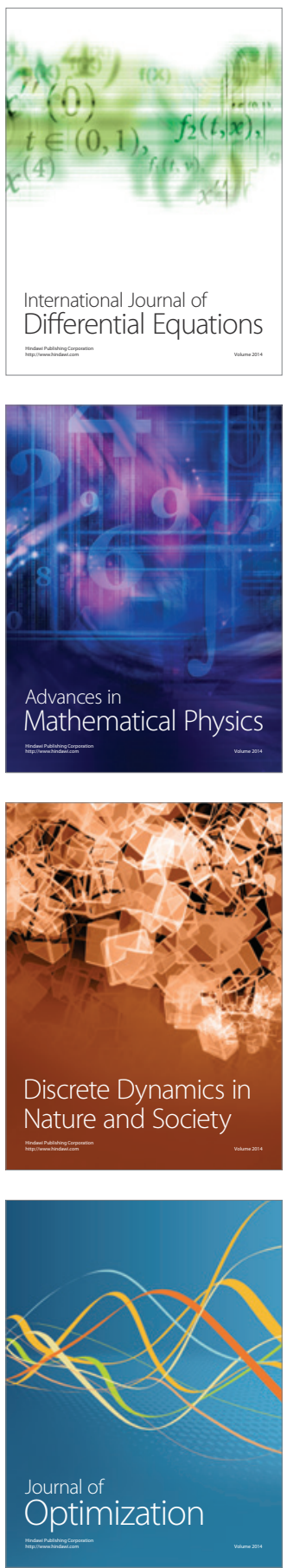